\title{
Frequency-shifting-based stable on-line algebraic parameter identification of linear systems
}

\author{
Josip Kasac*, Tihomir Zilic, Vladimir Milic, Andrej Jokic \\ University of Zagreb, \\ Faculty of Mechanical Engineering and Naval Architecture, \\ I. Lucica 5, HR-10000 Zagreb, Croatia
}

\begin{abstract}
In this paper a new approach to algebraic parameter identification of the linear SISO systems is proposed. The standard approach to the algebraic parameter identification is based on the algebraic derivatives in Laplace domain as the main tool for algebraic manipulations like elimination of the initial conditions and generation of linearly independent equations. This approach leads to the unstable time-varying state-space realization of the filters for the on-line parameter estimation. In this paper, the finite difference and shift operators in combination with the frequency-shifting property of Laplace transform is applied instead of algebraic derivatives. Resulting state-space realization of the estimator filters is asymptotically stable and doesn't require switch-of mechanism to prevent overflow of the estimator variables. The proposed method is especially suitable for applications in closed-loop on-line identification where the stable behavior of the estimators is a necessary requirement. The efficiency of the proposed algorithm is illustrated on three simulation examples.
\end{abstract}

Keywords: Algebraic identification; Parameter identification; Linear systems; Operational calculus

\section{Introduction}

There exist many different methods for parameter identification in the literature, like maximum likelihood methods [1], orthogonal functions [2], Kalman filtering [3] and leastsquares methods [4], among many others. Relatively recently, a new approach to parameter identification based on algebraic derivative method has been proposed by M. Fliess and H. Sira-Ramirez [5, 6] for fast and reliable parameter estimation in feedback control systems. The algebraic identification method provides an exact static formula for the unknown parameters which is based only on measurable input and output variables. The parameter

\footnotetext{
*Corresponding author

Email addresses: jkasac@fsb.hr (Josip Kasac), tzilic@fsb.hr (Tihomir Zilic), vmilic@fsb.hr (Vladimir Milic), ajokic@fsb.hr (Andrej Jokic)
} 
calculation expressions are obtained via algebraic manipulations based on derivative operator in operational domain $[7,8]$. This approach provides elimination of unknown initial conditions and generation of linear relations in the unknown parameters, so that accurate calculation of the parameters follows immediately from these relations. Unlike traditional methods, the obtained estimator is non-asymptotic: the convergence toward true values of the system parameters is quite fast (i.e., almost instantaneous). Also, the algebraic estimators do not need statistical knowledge of the measurement noise, neither it requires the classical persistency of excitation condition.

Several successful applications and experimental verifications of algebraic derivative approach have been reported in the literature. In [9, 10], an algebraic identification approach is used for the fast on-line determination of the parameters of sinusoidal signals with unknown amplitudes, phases and frequencies. A general algebraic framework for parameter estimation of signals described by differential equations is proposed in [11]. The algebraic derivative approach to state estimation is considered in $[12,13]$. In [14], the algebraic derivative method is applied for the derivative estimation of noisy signals. In [15, 16], an algebraic identification approach is proposed for linear systems with delayed inputs and structured perturbations.

For several applications of parameter and state estimation in feedback control systems the interested reader is referred to [17]. Next, several further reported applications are mentioned. In [18], the algebraic estimation algorithm is applied for the identification of the parameters of a permanent magnet stepper motor and a magnetic bearing. In [19], an online algebraic identification methodology for parameter and signal estimation in vibrating mechanical system is experimentally verified. The output control and simultaneous online algebraic parameter identification of perturbed 1-DOF suspension system is considered in [20]. In [21], an identification method for parameters estimation of a position-controlled servomechanism is proposed. In [22], a comparison between an algebraic parameter identification algorithm and classical asymptotic observers for the load of a boost converter is presented.

Despite the all mentioned advantages of algebraic parameter estimation method, there is a serious drawback, especially for applications in closed-loop on-line identification. Application of algebraic derivatives for elimination of initial conditions in combination with invariant filtering in the form of chain of integrators, leads to unstable time-varying state-space realization of estimator filters. Since the estimator variables are unbounded, additional switch off mechanism is necessary after a short period of time. Another disconnection strategy is based on introducing an extra auxiliary parameter, called the "sentinel" parameter that monitors the convergence of the rest of the estimated system parameters [20].

In this paper, an alternative approach to algebraic parameter identification, which provides stable on-line estimator filters realization, is proposed. The proposed approach is based on application of the finite difference and shift operator in operational domain, instead of algebraic derivative operator. Using these operators in combination with asymptotically stable invariant filtering, we obtain stable state-space realization of the estimator filters. In this way, the on-line parameter identification process doesn't require switch-off procedure after some short time, neither the use of "sentinel" variables. Furthermore, additional advantage 
of application of the shift operator is that state-space realization has lower dimension then in the case of using algebraic derivatives.

The remainder of the paper is organized as follows. Parameter identification problem formulation for linear time-invariant SISO systems is presented in Section 2. The algebraic parameter identification algorithm based on difference and shift operators is presented in Section 3. The simulation results are presented in Section 4. Finally, the concluding remarks are summarized in Section 5.

\section{Problem formulation}

\subsection{Continuous linear SISO system}

Consider the following continuous linear time-invariant system

$$
y^{(n)}(t)+\sum_{i=0}^{n-1} a_{i} y^{(i)}(t)=\sum_{j=0}^{m} b_{j} u^{(j)}(t),
$$

with unknown initial conditions

$$
y^{(i)}(0)=y_{0}^{(i)}, \quad i=0,1, \ldots, n-1,
$$

where $u(t) \in \mathbb{R}$ and $y(t) \in \mathbb{R}$ are the system input and output, respectively. The system order $n$ is a priori known, and $a_{0}, \ldots, a_{n-1}$ and $b_{0}, \ldots, b_{m}$ are unknown parameters.

Note that the problem of nonzero unknown initial conditions often occurs in practice, like for example in the identification of mechanical systems with weakly damped oscillations. Waiting that the system to reach zero steady-state in the identification experiment can be quite troublesome for the elastic systems with the small damping coefficients or for the processes with slow time constants [23].

The Laplace transform applied on the Eq. (1) gives the following expression in the operational domain

$$
s^{n} y(s)+\sum_{i=0}^{n-1} a_{i} s^{i} y(s)=\sum_{j=0}^{m} b_{j} s^{j} u(s)+\sum_{i=0}^{n-1} c_{i} s^{i},
$$

where $y(s)=\mathcal{L}\{y(t)\}$ and $u(s)=\mathcal{L}\{u(t)\}$ are Laplace transforms of $y(t)$ and $u(t)$, respectively. The coefficients $c_{i}$ on the right-side of Eq. (3) depend on unknown initial conditions (2). The Eq. (3) can be written as

$$
N(s) y(s)=B(s) u(s)+R(s)
$$

where

$$
N(s)=s^{n}+\sum_{i=0}^{n-1} a_{i} s^{i}, \quad B(s)=\sum_{j=0}^{m} b_{j} s^{j}, \quad R(s)=\sum_{i=0}^{n-1} c_{i} s^{i} .
$$


The main objective of this paper is on-line identification of the parameters $a_{0}, \ldots, a_{n-1}$ and $b_{0}, \ldots, b_{m}$ using only the measured input $u(t)$ and output $y(t)$, under assumption of unknown initial conditions. The second objective is determination of parameters $c_{0}, \ldots, c_{n-1}$.

The first step in the identification of unknown parameters $a_{0}, \ldots, a_{n-1}$ and $b_{0}, \ldots, b_{m}$ is the elimination of polynomial $R(s)$ with coefficients $c_{0}, \ldots, c_{n-1}$ which depend on unknown initial conditions (2). The second step is generation of a set of $n+m+1$ linearly independent algebraic equation for determination of $n+m+1$ parameters $a_{0}, \ldots, a_{n-1}$ and $b_{0}, \ldots, b_{m}$.

Instead of using algebraic derivative approach for elimination of initial conditions and generation of linearly independent equations, in this paper we apply the finite difference and shift operator in Laplace domain, which are presented in the following subsection.

\subsection{Finite difference operator in Laplace domain}

The finite difference operator in Laplace domain of a function $f(s)$ is defined as follows

$$
\delta_{q} f(s)=f(s+q)-f(s) .
$$

The operator $\delta_{q}$ can be represented as

$$
\delta_{q}=e^{q \frac{d}{d s}}-1
$$

where $e^{q \frac{d}{d s}}$ is the shift operator with property $e^{q \frac{d}{d s}} f(s)=f(s+q)$.

For $f(s)=s^{n}$, we have

$$
\delta_{q} s^{n}=(s+q)^{n}-s^{n}=\sum_{k=1}^{n}\left(\begin{array}{l}
n \\
k
\end{array}\right) s^{n-k} q^{k} .
$$

In other words, difference operator decreases the order of polynomials for one degree, so that $\delta_{q}^{n} s^{n-1}=0$, and

$$
\delta_{q}^{n} s^{k}=0, \quad \forall k<n,
$$

where $\delta_{q}^{n}$ denotes the $n$-th successive application of finite difference operator

$$
\delta_{q}^{n}=\left(e^{q \frac{d}{d s}}-1\right)^{n}=\sum_{k=0}^{n}(-1)^{k}\left(\begin{array}{l}
n \\
k
\end{array}\right) e^{(n-k) q \frac{d}{d s}} .
$$

For an arbitrary function $f(s)$ it follows that

$$
\delta_{q}^{n} f(s)=\sum_{k=0}^{n}(-1)^{k}\left(\begin{array}{l}
n \\
k
\end{array}\right) f(s+(n-k) q) .
$$

Applying the operator $\delta_{q}^{m}$ on function $f(s)=s^{n}$ for $m \leq n$, we get

$$
\delta_{q}^{m} s^{n}=\sum_{k=0}^{m}(-1)^{k}\left(\begin{array}{c}
m \\
k
\end{array}\right)(s+(m-k) q)^{n}=\sum_{k=0}^{m}(-1)^{k}\left(\begin{array}{c}
m \\
k
\end{array}\right) \sum_{j=0}^{n}\left(\begin{array}{l}
n \\
j
\end{array}\right) s^{n-j}(m-k)^{j} q^{j},
$$


or

$$
\delta_{q}^{m} s^{n}=\sum_{j=m}^{n} \gamma_{m j} s^{n-j}
$$

where

$$
\gamma_{m j}=q^{j}\left(\begin{array}{l}
n \\
j
\end{array}\right) \sum_{k=0}^{m}(-1)^{k}\left(\begin{array}{l}
m \\
k
\end{array}\right)(m-k)^{j} .
$$

The coefficient $\gamma_{m j}$ has the following property: $\gamma_{m j}=0$ for $j<m$ (triangular matrix structure).

Since the application of difference operator in Laplace domain produces shifted functions like $F(s+q)$, the frequency shifting property of Laplace transform

$$
F(s+q)=\mathcal{L}\left\{e^{-q t} f(t)\right\}
$$

will be used for the inverse transform of algebraic expressions to the time domain $[8,24]$.

\section{Algebraic parameter identification}

\subsection{Identification of parameters $a_{0}, \ldots, a_{n-1}$ and $b_{0}, \ldots, b_{m}$}

In this section we describe the main steps towards the estimation of parameters $a_{0}, \ldots, a_{n-1}$ and $b_{0}, \ldots, b_{m}$.

Step 1 - Elimination of initial conditions. The first step is annihilation of polynomial $R(s)$ which depends on unknown initial conditions. Since the degree of the polynomial $R(s)$ is $\operatorname{deg}(R)=n-1$, it follows from $(9)$ that $\delta_{q}^{n} R(s)=0$. Applying the operator $\delta_{q}^{n}$ on $(3)$, the following expression is obtained

$$
\bar{y}_{n}(s)+\sum_{i=0}^{n-1} a_{i} \bar{y}_{i}(s)=\sum_{j=0}^{m} b_{j} \bar{u}_{j}(s)
$$

where

$$
\begin{aligned}
& \bar{y}_{i}(s)=\delta_{q}^{n}\left[s^{i} y(s)\right]=\sum_{k=0}^{n}(-1)^{k}\left(\begin{array}{l}
n \\
k
\end{array}\right)(s+(n-k) q)^{i} y(s+(n-k) q), \\
& \bar{u}_{j}(s)=\delta_{q}^{n}\left[s^{j} u(s)\right]=\sum_{k=0}^{n}(-1)^{k}\left(\begin{array}{l}
n \\
k
\end{array}\right)(s+(n-k) q)^{j} u(s+(n-k) q) .
\end{aligned}
$$

Step 2 - Generation of linearly independent equations. The next step is generation of $n+m+1$ linearly independent equation from Eq. (16). This can be done using the shift operator $e^{q \frac{d}{d s}}$.

Applying the operator $e^{l q \frac{d}{d s}}$ for $l=0,1, \ldots, n+m$, on (16), the following set of algebraic equations is obtained

$$
\bar{y}_{n}(s+l q)+\sum_{i=0}^{n-1} a_{i} \bar{y}_{i}(s+l q)=\sum_{j=0}^{m} b_{j} \bar{u}_{j}(s+l q), \quad l=0,1, \ldots, n+m,
$$


or

$$
\bar{Y}_{l n}(s)+\sum_{i=0}^{n-1} a_{i} \bar{Y}_{l i}(s)=\sum_{j=0}^{m} b_{j} \bar{U}_{l j}(s), \quad l=0,1, \ldots, n+m,
$$

where the following notation is introduced

$$
\begin{aligned}
& \bar{Y}_{l i}(s)=\bar{y}_{i}(s+l q)=\sum_{k=0}^{n}(-1)^{k}\left(\begin{array}{l}
n \\
k
\end{array}\right)(s+(n+l-k) q)^{i} y(s+(n+l-k) q), \\
& \bar{U}_{l j}(s)=\bar{u}_{j}(s+l q)=\sum_{k=0}^{n}(-1)^{k}\left(\begin{array}{l}
n \\
k
\end{array}\right)(s+(n+l-k) q)^{j} u(s+(n+l-k) q),
\end{aligned}
$$

for $i=0,1, \ldots, n, j=0,1, \ldots, m, l=0,1, \ldots, n+m$. After the change of indices $p=n+l-k$, the above expressions become

$$
\bar{Y}_{l i}(s)=\sum_{p=l}^{n+l} \mu_{l p}(s+p q)^{i} y(s+p q), \quad \bar{U}_{l j}(s)=\sum_{p=l}^{n+l} \mu_{l p}(s+p q)^{j} u(s+p q),
$$

where we have used the following shorthand notation

$$
\mu_{l p}=(-1)^{n+l-p}\left(\begin{array}{c}
n \\
n+l-p
\end{array}\right)
$$

for $l \leq p \leq n+l$.

Step 3 - Invariant filtering. In order to overcome effects of high-frequency noise in the measurement of the output variable we must avoid time-derivatives of output variable, which are represented by terms $s^{i} y(s)=\mathcal{L}\left\{y^{(i)}(t)\right\}$ in Eq. (23). Multiplying both sides of Eq. (20) by the transfer function

$$
G(s)=\frac{1}{D(s)}, \quad D(s)=\prod_{k=1}^{\bar{n}}\left(s+\bar{q}_{k}\right)
$$

which represents a low-pass filter, where $\bar{n}>n, \bar{q}_{k}>0$ and $\bar{q}_{k} \neq p q$ for $k=1, \ldots, \bar{n}$ and $p=0,1, \ldots, 2 n+m$, the following expression is obtained

$$
Y_{l n}(s)+\sum_{i=0}^{n-1} a_{i} Y_{l i}(s)=\sum_{j=0}^{m} b_{j} U_{l j}(s)
$$

where

$$
Y_{l i}(s)=\sum_{p=l}^{n+l} \mu_{l p} \frac{(s+p q)^{i}}{D(s)} y(s+p q), \quad U_{l j}(s)=\sum_{p=l}^{n+l} \mu_{l p} \frac{(s+p q)^{j}}{D(s)} u(s+p q) .
$$


By using the method of partial fraction, it follows that

$$
\begin{aligned}
& \tilde{y}_{p i}(s)=\frac{(s+p q)^{i}}{D(s)} y(s+p q)=\sum_{k=1}^{\bar{n}} \frac{A_{p i k}}{s+\bar{q}_{k}} y(s+p q), \\
& \tilde{u}_{p j}(s)=\frac{(s+p q)^{j}}{D(s)} u(s+p q)=\sum_{k=1}^{\bar{n}} \frac{A_{p j k}}{s+\bar{q}_{k}} u(s+p q),
\end{aligned}
$$

where the coefficients $A_{p i k}$ are determined by

$$
A_{p i k}=\lim _{s \rightarrow-\bar{q}_{k}}\left(s+\bar{q}_{k}\right) \frac{(s+p q)^{i}}{D(s)}=\frac{\left(p q-\bar{q}_{k}\right)^{i}}{\prod_{j=1 ; j \neq k}^{\bar{n}}\left(\bar{q}_{j}-\bar{q}_{k}\right)} .
$$

By defining the state variables in operational domain

$$
x_{p k}(s)=\frac{y(s+p q)}{s+\bar{q}_{k}}, \quad w_{p k}(s)=\frac{u(s+p q)}{s+\bar{q}_{k}},
$$

the expressions (28) and (29) can be rewritten as

$$
\tilde{y}_{p i}(s)=\sum_{k=1}^{\bar{n}} A_{p i k} x_{p k}(s), \quad \tilde{u}_{p j}(s)=\sum_{k=1}^{\bar{n}} A_{p j k} w_{p k}(s),
$$

and finally

$$
Y_{l i}(s)=\sum_{p=l}^{n+l} \mu_{l p} \tilde{y}_{p i}(s), \quad U_{l j}(s)=\sum_{p=l}^{n+l} \mu_{l p} \tilde{u}_{p j}(s) .
$$

Step 4 - State-space realization. The following step is the state-space realization which provides on-line parameter identification. In the time domain the expressions in Eq. (31) become a set of stable decoupled linear differential equations

$$
\dot{x}_{p k}(t)+\bar{q}_{k} x_{p k}(t)=e^{-p q t} y(t), \quad \dot{w}_{p k}(t)+\bar{q}_{k} w_{p k}(t)=e^{-p q t} u(t),
$$

with zero initial conditions, and expressions in Eq. (33) become

$$
Y_{l i}(t)=\sum_{p=l}^{n+l} \mu_{l p} \sum_{k=1}^{\bar{n}} A_{p i k} x_{p k}(t), \quad U_{l j}(t)=\sum_{p=l}^{n+l} \mu_{l p} \sum_{k=1}^{\bar{n}} A_{p j k} w_{p k}(t) .
$$

Furthermore, in the time domain Eq. (26) becomes

$$
Y_{l n}(t)+\sum_{i=0}^{n-1} a_{i} Y_{l i}(t)=\sum_{j=0}^{m} b_{j} U_{l j}(t)
$$


By introducing vectors $\mathbf{y}_{n}(t)=\left[\begin{array}{lllll}Y_{0 n}(t) & Y_{1 n}(t) & \cdots & Y_{(n+m) n}(t)\end{array}\right]^{T}, \mathbf{a}=\left[\begin{array}{llll}a_{0} & a_{1} & \cdots & a_{n-1}\end{array}\right]^{T}$, $\mathbf{b}=\left[\begin{array}{llll}b_{0} & b_{1} & \cdots & b_{m}\end{array}\right]^{T}$, and matrices $\mathbf{Y}(t) \in \mathbb{R}^{(n+m+1) \times n}, \mathbf{U}(t) \in \mathbb{R}^{(n+m+1) \times(m+1)}$ with elements $Y_{l i}(t)$ and $U_{l j}(t)$ respectively, the expression (36) can be rewritten as follows

$$
\mathbf{y}_{n}(t)=-\mathbf{Y}(t) \mathbf{a}+\mathbf{U}(t) \mathbf{b}=\left[\begin{array}{ll}
-\mathbf{Y}(t) & \mathbf{U}(t)
\end{array}\right]\left[\begin{array}{l}
\mathbf{a} \\
\mathbf{b}
\end{array}\right]=\boldsymbol{\Phi}(t) \boldsymbol{\theta},
$$

where $\boldsymbol{\Phi}(t)=[-\mathbf{Y}(t) \mathbf{U}(t)]$ and $\boldsymbol{\theta}=\left[\begin{array}{ll}\mathbf{a}^{T} & \mathbf{b}^{T}\end{array}\right]^{T}$.

Since the differential equations (34) are asymptotically stable and the inputs $y(t)$ and $u(t)$ are multiplied with decreasing in time exponential functions, it follows that solutions $x_{p k}(t)$ and $w_{p k}(t)$ will be bounded even for unbounded inputs which satisfy: $|y(t)| \leq \alpha_{1} e^{q t}$ and $|u(t)| \leq \alpha_{2} e^{q t}$, for some $\alpha_{1}, \alpha_{2}>0$. In other words, the elements of matrix $\boldsymbol{\Phi}(t)$ and vector $\mathbf{y}_{n}(t)$ will be bounded for every $t>0$.

Step 5 - Singularity-free parameters calculation. The vector of unknown parameters $\boldsymbol{\theta}$ can be obtained from (37)

$$
\boldsymbol{\theta}=\boldsymbol{\Phi}^{-1}(t) \mathbf{y}_{n}(t)=\frac{\operatorname{adj}(\boldsymbol{\Phi}(t))}{\operatorname{det}(\boldsymbol{\Phi}(t))} \mathbf{y}_{n}(t)=\frac{\boldsymbol{\varphi}(t)}{\operatorname{det}(\boldsymbol{\Phi}(t))},
$$

where $\boldsymbol{\varphi}(t)=\operatorname{adj}(\boldsymbol{\Phi}(t)) \mathbf{y}_{n}(t)=\left[\begin{array}{llll}\varphi_{1}(t) & \varphi_{2}(t) & \cdots & \varphi_{n+m+1}(t)\end{array}\right]^{T}$. The system is said to be identifiable if, and only if, $\operatorname{det}(\boldsymbol{\Phi}(t)) \neq 0,[5,25]$.

The problem with the expression (38) is that at time $t=0$, both the numerator and the denominator are zero. Since the quotient is undetermined, we must evaluate the formula starting at a later time $t \geq \varepsilon>0$.

The determinant that belongs to the identifier denominator may still cross the singular value of zero, causing a local loss of identifiability. An approach to avoid this problem is multiplication of equation $(37)$ by $\boldsymbol{\Phi}^{T}(t)$ and integration during the time interval $[0, t]$, to obtain

$$
\int_{0}^{t} \boldsymbol{\Phi}^{T}(\tau) \mathbf{y}_{n}(\tau) d \tau=\left(\int_{0}^{t} \boldsymbol{\Phi}^{T}(\tau) \boldsymbol{\Phi}(\tau) d \tau\right) \boldsymbol{\theta}
$$

Since the matrix

$$
\mathbf{S}(t)=\int_{0}^{t} \boldsymbol{\Phi}^{T}(\tau) \boldsymbol{\Phi}(\tau) d \tau
$$

is symmetric and positive definite it is invertible, and linear system of equations (39) can be solved for $t \geq \varepsilon>0$ using efficient numerical method like LU or Cholesky decomposition $[18]$.

Fig. 1 illustrates the above described steps in derivation of the final parameter identification algorithm, which is further schematically illustrated in Fig. 2.

\subsection{Identification of parameters $c_{0}, c_{1}, \ldots, c_{n-1}$}

The identification of parameters $c_{0}, c_{1}, \ldots, c_{n-1}$ follows after the coefficients $a_{0}, \ldots, a_{n-1}$, $b_{0}, \ldots, b_{m}$ have been determined. The procedure for this calculation is derived and presented next. 


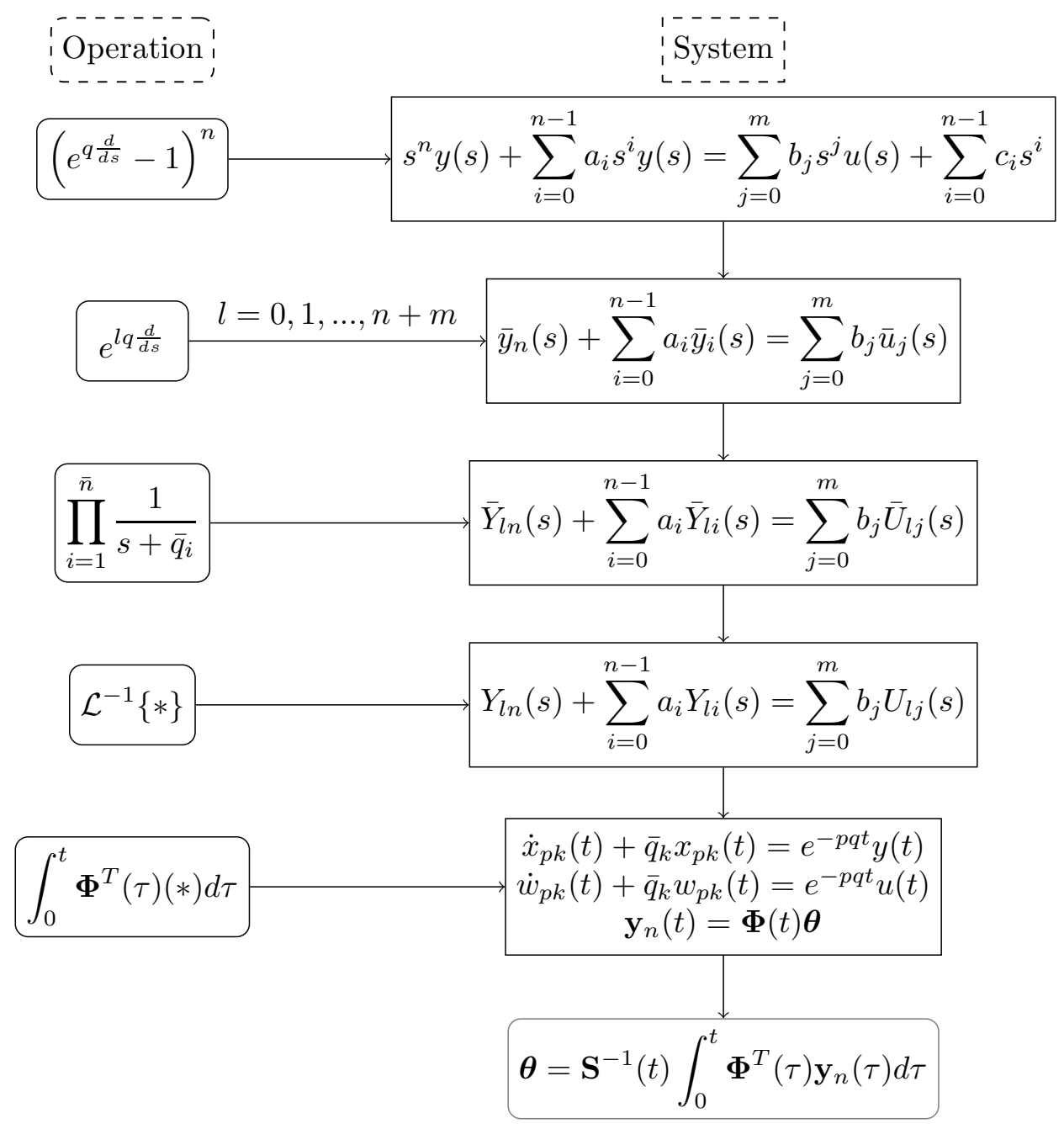

Figure 1: The schematic illustration of the main steps for the algorithm derivation. 


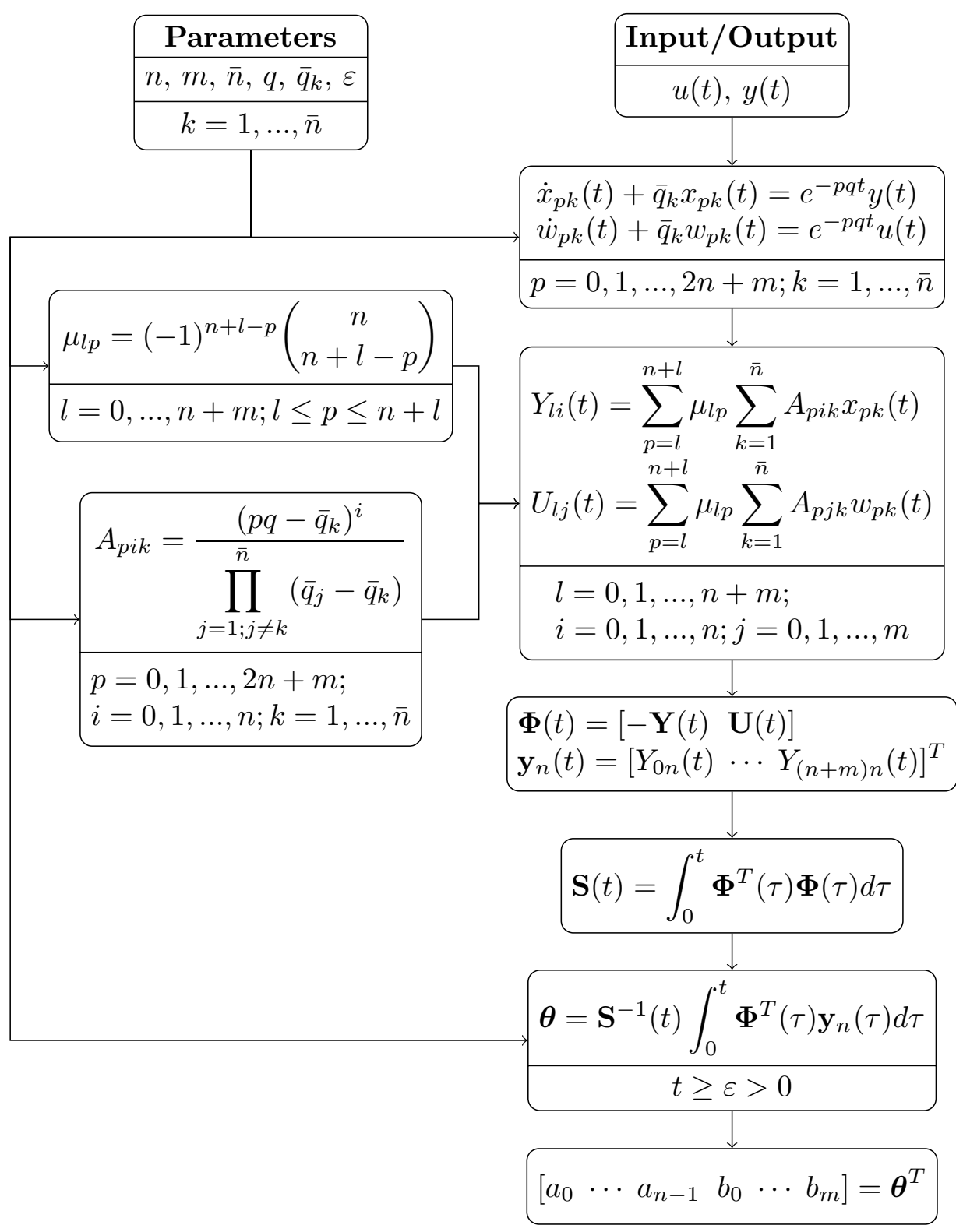

Figure 2: The schematic illustration of the final parameter identification algorithm. 
If we apply the operator $\delta_{q}^{l}$ for $l=0,1, \ldots, n-1$ on $(3)$, we get

$$
\delta_{q}^{l}\left[s^{n} y(s)\right]+\sum_{i=0}^{n-1} a_{i} \delta_{q}^{l}\left[s^{i} y(s)\right]-\sum_{j=0}^{m} b_{j} \delta_{q}^{i}\left[s^{j} u(s)\right]=\sum_{i=l}^{n-1} c_{i} \delta_{q}^{l} s^{i}
$$

where

$$
\begin{gathered}
\delta_{q}^{l}\left[s^{i} y(s)\right]=\sum_{k=0}^{l}(-1)^{k}\left(\begin{array}{l}
l \\
k
\end{array}\right)(s+(l-k) q)^{i} y(s+(l-k) q), \\
\delta_{q}^{l}\left[s^{j} u(s)\right]=\sum_{k=0}^{l}(-1)^{k}\left(\begin{array}{l}
l \\
k
\end{array}\right)(s+(l-k) q)^{j} u(s+(l-k) q), \\
\delta_{q}^{l} s^{i}=\sum_{k=l}^{i} \gamma_{l k} s^{i-k},
\end{gathered}
$$

for $i=0,1, \ldots, n-1$ and $j=0,1, \ldots, m$.

By changing index notation $p=l-k$, the above mentioned expressions become

$$
\delta_{q}^{l}\left[s^{i} y(s)\right]=\sum_{p=0}^{l} \eta_{l p}(s+p q)^{i} y(s+p q), \quad \delta_{q}^{l}\left[s^{j} u(s)\right]=\sum_{p=0}^{l} \eta_{l p}(s+p q)^{j} u(s+p q),
$$

where

$$
\eta_{l p}=(-1)^{l-p}\left(\begin{array}{c}
l \\
l-p
\end{array}\right)
$$

By multiplying equation (41) with transfer function (25) we get

$$
\hat{Y}_{l n}(s)+\sum_{i=0}^{n-1} a_{i} \hat{Y}_{l i}(s)-\sum_{j=0}^{m} b_{j} \hat{U}_{l j}(s)=\sum_{i=l}^{n-1} c_{i} Q_{l i}(s),
$$

where

$$
\hat{Y}_{l i}(s)=\sum_{p=0}^{l} \eta_{l p} \frac{(s+p q)^{i}}{D(s)} y(s+p q), \quad \hat{U}_{l j}(s)=\sum_{p=0}^{l} \eta_{l p} \frac{(s+p q)^{j}}{D(s)} u(s+p q)
$$

and

$$
Q_{l i}(s)=\sum_{k=l}^{i} \gamma_{l k} \frac{s^{i-k}}{D(s)}
$$

By introducing change of indices $p=i-k$, the equation (49) becomes

$$
Q_{l i}(s)=\sum_{k=l}^{i} \gamma_{l k} \frac{s^{i-k}}{D(s)}=\sum_{p=0}^{i-l} \gamma_{l, i-p} \frac{s^{p}}{D(s)} .
$$


By using the method of partial fraction, it follows that

$$
\frac{s^{p}}{D(s)}=\sum_{j=1}^{\bar{n}} \frac{B_{p j}}{s+\bar{q}_{j}}
$$

where

$$
B_{p j}=\lim _{s \rightarrow-\bar{q}_{j}}\left(s+\bar{q}_{j}\right) \frac{s^{p}}{D(s)}=\frac{\left(-\bar{q}_{j}\right)^{p}}{\prod_{i=1 ; i \neq j}^{\bar{n}}\left(\bar{q}_{i}-\bar{q}_{j}\right)}
$$

so that

$$
Q_{l i}(s)=\sum_{p=0}^{i-l} \gamma_{l, i-p} \sum_{j=1}^{\bar{n}} \frac{B_{p j}}{s+\bar{q}_{j}} .
$$

In time domain, the expression (47) becomes

$$
\hat{Y}_{l n}(t)+\sum_{i=0}^{n-1} a_{i} \hat{Y}_{l i}(t)-\sum_{j=0}^{m} b_{j} \hat{U}_{l j}(t)=\sum_{i=l}^{n-1} c_{i} Q_{l i}(t),
$$

where

$$
\hat{Y}_{l i}(t)=\sum_{p=0}^{l} \eta_{l p} \sum_{k=1}^{\bar{n}} A_{p i k} x_{p k}(t), \quad \hat{U}_{l j}(t)=\sum_{p=0}^{l} \eta_{l p} \sum_{k=1}^{\bar{n}} A_{p j k} w_{p k}(t),
$$

and

$$
Q_{l i}(t)=\sum_{p=0}^{i-l} \gamma_{l, i-p} \sum_{j=1}^{\bar{n}} B_{p j} e^{-\bar{q}_{j} t} .
$$

By introducing vectors $\mathbf{c}=\left[\begin{array}{lllll}c_{0} & c_{1} & \cdots & c_{n-1}\end{array}\right]^{T}, \hat{\mathbf{y}}_{n}(t)=\left[\begin{array}{llll}\hat{Y}_{0 n}(t) & \hat{Y}_{1 n}(t) & \cdots & Y_{(n-1) n}(t)\end{array}\right]^{T}$, and matrices $\hat{\mathbf{Y}}(t) \in \mathbb{R}^{n \times n}, \hat{\mathbf{U}}(t) \in \mathbb{R}^{n \times(m+1)}$ and $\mathbf{Q}(t) \in \mathbb{R}^{n \times n}$ with elements $\hat{Y}_{l i}(t), \hat{U}_{l i}(t)$ and $Q_{l i}(t)$ respectively, the expression (54) can be rewritten as follows

$$
\hat{\mathbf{y}}_{n}(t)+\hat{\mathbf{Y}}(t) \mathbf{a}-\hat{\mathbf{U}}(t) \mathbf{b}=\mathbf{Q}(t) \mathbf{c},
$$

so that

$$
\mathbf{c}=\mathbf{Q}^{-1}(t)\left(\hat{\mathbf{y}}_{n}(t)-\hat{\boldsymbol{\Phi}}(t) \boldsymbol{\theta}\right)
$$

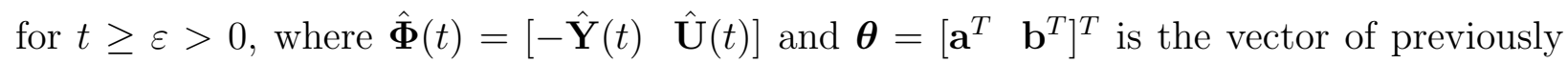
estimated parameters. The singularity-free calculation of the parameter vector $\mathbf{c}$ can be done using the same approach as in the case of the parameter vector $\boldsymbol{\theta}$, as presented in Section 3.1. 


\section{Simulation Examples}

The performances of the proposed algebraic parameter identification algorithm will be illustrated on the three examples related to the on-line parameter identification of the vibrating mechanical system with exogenous perturbation.

The dynamical model of the vibrating mechanical system is described by the differential equation

$$
m \ddot{y}(t)+c \dot{y}(t)+k y(t)=u(t)+f(t),
$$

where $y(t)$ is the mass displacement, $u(t)$ is the control input (force), and $f(t)$ is an external disturbance. The unknown system parameters are the mass $m$, viscous damping $c$ and stiffness constant $k$.

\subsection{Example 1: Frequency estimation of the 1-DOF mechanical oscillator}

Consider first the mechanical system with known mass $m=1 \mathrm{~kg}$, without dissipation $(c=0)$ and control input $(u(t)=0)$, with constant unknown external force, $f(t)=F_{0}$, and with unknown initial conditions $y(0)=y_{0}$ and $\dot{y}(0)=v_{0}$,

$$
\ddot{y}(t)+k y(t)=F_{0}, \quad y(0)=y_{0}, \quad \dot{y}(0)=v_{0} .
$$

The problem is on-line identification of the stiffness coefficient $k$ in the case when the constant external force and initial conditions are unknown, and the displacement measurement $y(t)$ is only available.

The Laplace transform of the differential equation (60) is

$$
s^{2} y(s)-s y_{0}-v_{0}+k y(s)=\frac{F_{0}}{s} .
$$

Multiplying out the expression (61) by the complex frequency $s$, we obtain after rearrangement

$$
s^{3} y(s)+k s y(s)=c_{2} s^{2}+c_{1} s+c_{0},
$$

where $c_{2}=y_{0}, c_{1}=v_{0}$ and $c_{0}=F_{0}$.

Note that the general solution of the system (60) is

$$
y(t)=A \sin (\omega t+\phi)+K,
$$

where $\omega=\sqrt{k}, K=F_{0} / k$, and the amplitude $A$ and the phase $\phi$ depend on the initial conditions. Taking the Laplace transform of the solution (63), we obtain after rearrangement the same expression as (62) with the coefficients $c_{2}=K+A \sin (\phi), c_{1}=A \omega \cos (\phi)$ and $c_{0}=\omega^{2} K$.

In other words, the identification of the stiffness coefficient $k$ of the system (60) with unknown initial conditions and constant external force is completely equivalent problem as the frequency estimation of the periodic signal (63) with unknown bias, amplitude and phase. This problem is solved in [9] by using the standard algebraic derivative method (ADM). In the remainder of this subsection we will compare the performances of the frequency-shifting method (FSM) presented in the previous section with the ADM presented in [9]. 


\subsubsection{Algebraic derivative method (ADM)}

The first step in the algebraic approach to the parameter identification is elimination of the unknown coefficients $c_{0}, c_{1}$ and $c_{2}$ on the right-hand side of the expression (62). The ADM uses derivative operator with respect to complex frequency $s$ for the elimination of the right-hand side of the Eq. (62). By differentiating the expression (62) three times with respect to the complex variable $s$, the following expression is obtained

$$
s^{3} \frac{d^{3} y(s)}{d s^{3}}+9 s^{2} \frac{d^{2} y(s)}{d s^{2}}+18 s \frac{d y(s)}{d s}+6 y(s)+k\left[s \frac{d^{3} y(s)}{d s^{3}}+3 \frac{d^{2} y(s)}{d s^{2}}\right]=0,
$$

which is free of the unknown coefficients $c_{0}, c_{1}$ and $c_{2}$.

By multiplying the expression (64) with $s^{-3}$, representing three iterated integrations in the time domain, it is obtained the following expression

$$
\frac{d^{3} y(s)}{d s^{3}}+9 \frac{1}{s} \frac{d^{2} y(s)}{d s^{2}}+18 \frac{1}{s^{2}} \frac{d y(s)}{d s}+6 \frac{1}{s^{3}} y(s)+k\left[\frac{1}{s^{2}} \frac{d^{3} y(s)}{d s^{3}}+3 \frac{1}{s^{3}} \frac{d^{2} y(s)}{d s^{2}}\right]=0,
$$

which is free of terms containing positive powers of the complex variable $s$, representing undesired repeated time differentiations of the involved signals.

In the time domain, the expression (65) can be written as: $-n(t)+k d(t)=0$, where

$$
\begin{array}{ll}
n(t)=z_{1}+t^{3} y(t), & d(t)=z_{4}, \\
\dot{z}_{1}=z_{2}-9 t^{2} y(t), & \dot{z}_{4}=z_{5}, \\
\dot{z}_{2}=z_{3}+18 t y(t), & \dot{z}_{5}=z_{6}-t^{3} y(t), \\
\dot{z}_{3}=6 y(t), & \dot{z}_{6}=3 t^{2} y(t) .
\end{array}
$$

Note that the Eq. (66) is the unstable time-varying linear system in perturbed Brunovsky's form. The output variables $n(t)$ and $d(t)$ are additionally filtered using the same second order low-pass filter

$$
\ddot{n}_{f}=-2 \zeta \omega_{n} \dot{n}_{f}-\omega_{n}^{2}\left(n_{f}-n(t)\right), \quad \ddot{d}_{f}=-2 \zeta \omega_{n} \dot{d}_{f}-\omega_{n}^{2}\left(d_{f}-d(t)\right),
$$

where $\omega_{n}$ is the cut-off frequency and $\zeta$ is the damping factor. From the state-space realization (66) and (67) it follows that the ADM-based estimator filter is of the 10th order.

Since the quotient $k=n(t) / d(t)$ is not affected by the invariant filtering (67), the final estimate is

$$
k=\frac{n_{f}(t)}{d_{f}(t)} .
$$

In order to avoid that the denominator $d_{f}(t)$ cross the singular value of zero, an additional invariant nonlinear filtering is proposed in [20]. By taking the integral of the absolute value of the numerator and denominator, the fraction holds invariant

$$
k=\left(\int_{0}^{t}\left|n_{f}(\tau)\right| d \tau\right)\left(\int_{0}^{t}\left|d_{f}(\tau)\right| d \tau\right)^{-1}
$$

and the denominator of Eq. (69) is always strictly positive for $t \geq \varepsilon>0$.

The following parameters of the ADM-based estimator are used in the simulation: the cut-off frequency $\omega_{n}=15 \mathrm{rad} / \mathrm{s}$, and the damping factor $\zeta=0.707$. 


\subsubsection{Frequency-shifting method (FSM)}

By comparing (62) with (1) it follows that $a_{2}=0, a_{1}=k$ and $a_{0}=0$, so that Eq. (26) becomes

$$
Y_{03}(s)+a_{1} Y_{01}(s)=0
$$

where, based on Eq. (27) and (28), it follows

$$
Y_{0 i}(s)=\sum_{p=0}^{3} \mu_{0 p} \frac{(s+p q)^{i}}{D(s)} y(s+p q)=\sum_{p=0}^{3} \mu_{0 p} \sum_{k=1}^{\bar{n}} \frac{A_{p i k}}{s+\bar{q}_{k}} y(s+p q), \quad i=1,3 .
$$

By interchanging the summations in the above expression, it follows

$$
Y_{0 i}(s)=\sum_{k=1}^{\bar{n}} z_{i k}(s), \quad z_{i k}(s)=\frac{1}{s+\bar{q}_{k}} \sum_{p=0}^{3} \mu_{0 p} A_{p i k} y(s+p q), \quad i=1,3 .
$$

In the time domain, expressions in (72) become

$$
Y_{0 i}(t)=\sum_{k=1}^{\bar{n}} z_{i k}(t), \quad \dot{z}_{i k}+\bar{q}_{k} z_{i k}=y(t) \sum_{p=0}^{3} \mu_{0 p} A_{p i k} e^{-p q t}
$$

for $i=1,3$ and $k=1, \ldots, \bar{n}$. Note that the Eq. (73) defines a stable time-varying linear system in the Jordan canonical form. By taking $\bar{n}=5$, the state-space realization (73) of the FSM-based estimator filter is also of the 10th order, same as the dimension of the ADM-based estimator filter.

Finally, in the time domain from the Eq. (70) it follows

$$
k=-\frac{Y_{03}(t)}{Y_{01}(t)} .
$$

The singularity free solution is calculated using Eq. (69), by replacing $n_{f}(t)$ with $-Y_{03}(t)$ and $d_{f}(t)$ with $Y_{01}(t)$.

The following parameters of the FSM-based estimator are used in the simulation: frequency shift $q=0.5$, and filter poles are $s_{k}=-\bar{q}_{k}=-(4+k)$, for $k=1, \ldots, 5$.

\subsubsection{Comparison of $A D M$ and FSM}

The parameters of the output signal (63) used in simulations are: $A=0.2 \mathrm{~m}, K=0.1$ $\mathrm{m}, \phi=1 \mathrm{rad}$ and $\omega=5 \mathrm{rad} / \mathrm{s}$. The sampling time is $0.002 \mathrm{~s}$.

Fig. 3 shows the output signal without measurement noise and estimated parameter $k=\omega^{2}$ calculated by the ADM and FSM-based estimators. We can see that in the time instant $\varepsilon=1 \mathrm{~s}$ when the estimators are switched on, the convergence toward real values of the parameter $k$ is almost instantaneous. By comparing the absolute relative error (ARE) defined by

$$
A R E(t)=\left|\frac{k(t)-k_{\text {real }}}{k_{\text {real }}}\right|
$$


where $k(t)$ and $k_{\text {real }}$ are the estimated and real value of the parameter, we can observe that the FSM-based estimator provides faster convergence toward the real values of the parameter $k$.

Fig. 4 shows the numerator and denominator in expressions (68) and (74) for ADM and FSM-based estimators. Since the ADM-based estimator (66) is unstable, $n(t)$ and $d(t)$ growing unboundedly in time. In the case of the FSM-based estimator numerator and denominator are bounded in time.

Since the measurement noise and sampling period significantly affect the performances of the parameter estimation algorithms, several simulations are performed in order to compare the robustness of the ADM and FSM-based estimators. The measured signal $y_{m}(t)=$ $y(t)+\xi(t)$ is the output sinal $y(t)$ perturbed with the additive zero mean uniform noise $\xi(t)$. The amount of additive noise affecting the signal is usually expressed by the signal-to-noise ratio (SNR). The SNR is defined as the ratio of the signal power to the noise power,

$$
S N R=\frac{P_{\text {signal }}}{P_{\text {noise }}}=\left(\frac{1}{T} \int_{0}^{T} y^{2}(t) d t\right)\left(\frac{1}{T} \int_{0}^{T} \xi^{2}(t) d t\right)^{-1},
$$

where $T$ is the time interval of the signal. The SNR is usually expressed in decibels, $S N R(d B)=10 \log _{10}(S N R)$.

Fig. 5 illustrates the convergence of the ADM and FSM-based estimators for different values of signal-to-noise rate $S N R$ and sampling time $h$. It can be seen that, comparing with the FSM-based estimator, the estimation error in the case of the ADM-based estimator becomes larger and more non-stationary for the larger values of the sampling time and for the smaller values of the $S N R$.

The estimator performances depending on the $S N R$ and $h$ are more precisely illustrated in Fig. 6. The error is measured by the mean absolute percentage error (MAPE) [10], defined in the time interval $[\varepsilon, T]$ by

$$
M A P E=\frac{100}{T-\varepsilon} \int_{\varepsilon}^{T} A R E(t) d t .
$$

The average MAPE based on 300 Monte Carlo simulations is obtained for each value of

$S N R$. The average values of $M A P E$ for signals with small amount of noise $(S N R=23.62$ $\mathrm{dB})$ and large amount of noise $(S N R=3.48 \mathrm{~dB})$, for ADM and FSM-based estimators, are listed in Table 1. From the Fig. 6 we can see that difference in MAPE between ADM and FSM-based estimators is larger as $S N R$ decreases and $h$ increases. In the other words, the FSM-based estimator is more robust to the measurement noise and values of the sampling time then the ADM-based estimator.

\subsection{Example 2: Parameters identification of the 1-DOF mechanical system}

As the second example we consider the FSM-based parameter identification of the unperturbed mechanical system (59) when $f(t)=0$, where only measurements of the displacement $y(t)$ and the control input $u(t)$ are available. The nominal values of the system parameters for the numerical simulations are $m=1.8754 \mathrm{~kg}, c=3.7367 \mathrm{Ns} / \mathrm{m}, k=377.2706 \mathrm{~N} / \mathrm{m}$ and 

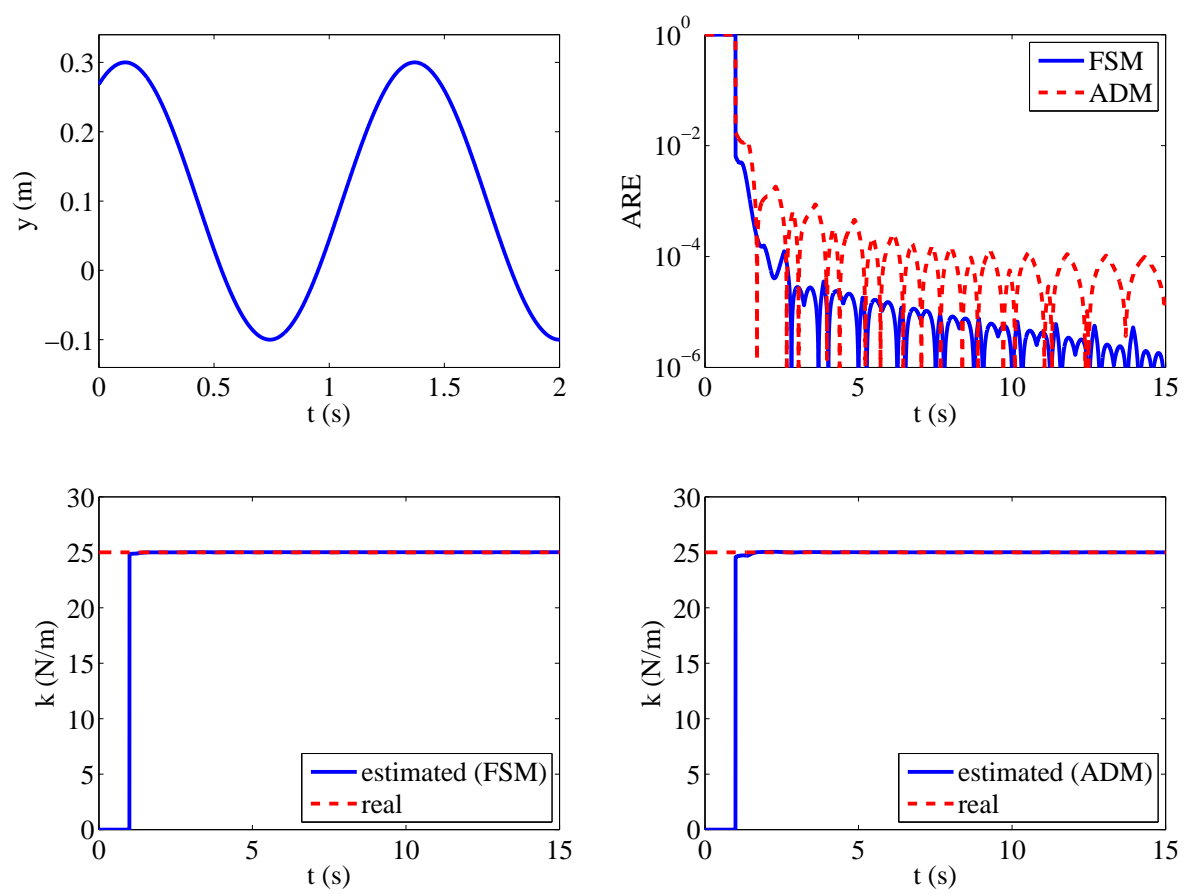

Figure 3: The comparison of ADM and FSM-based estimators in the case without measurement noise.
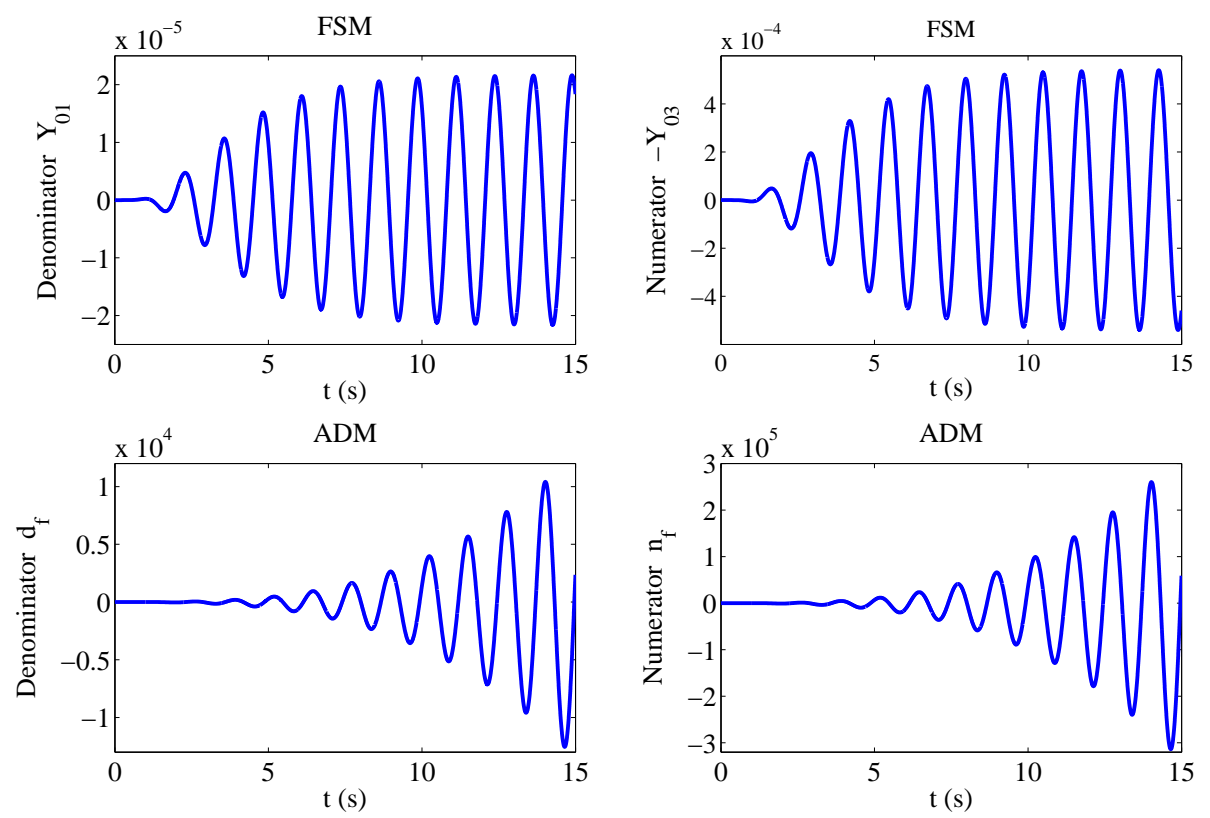

Figure 4: The numerator and denominator in expressions (68) and (74) for ADM and FSM-based estimators. 

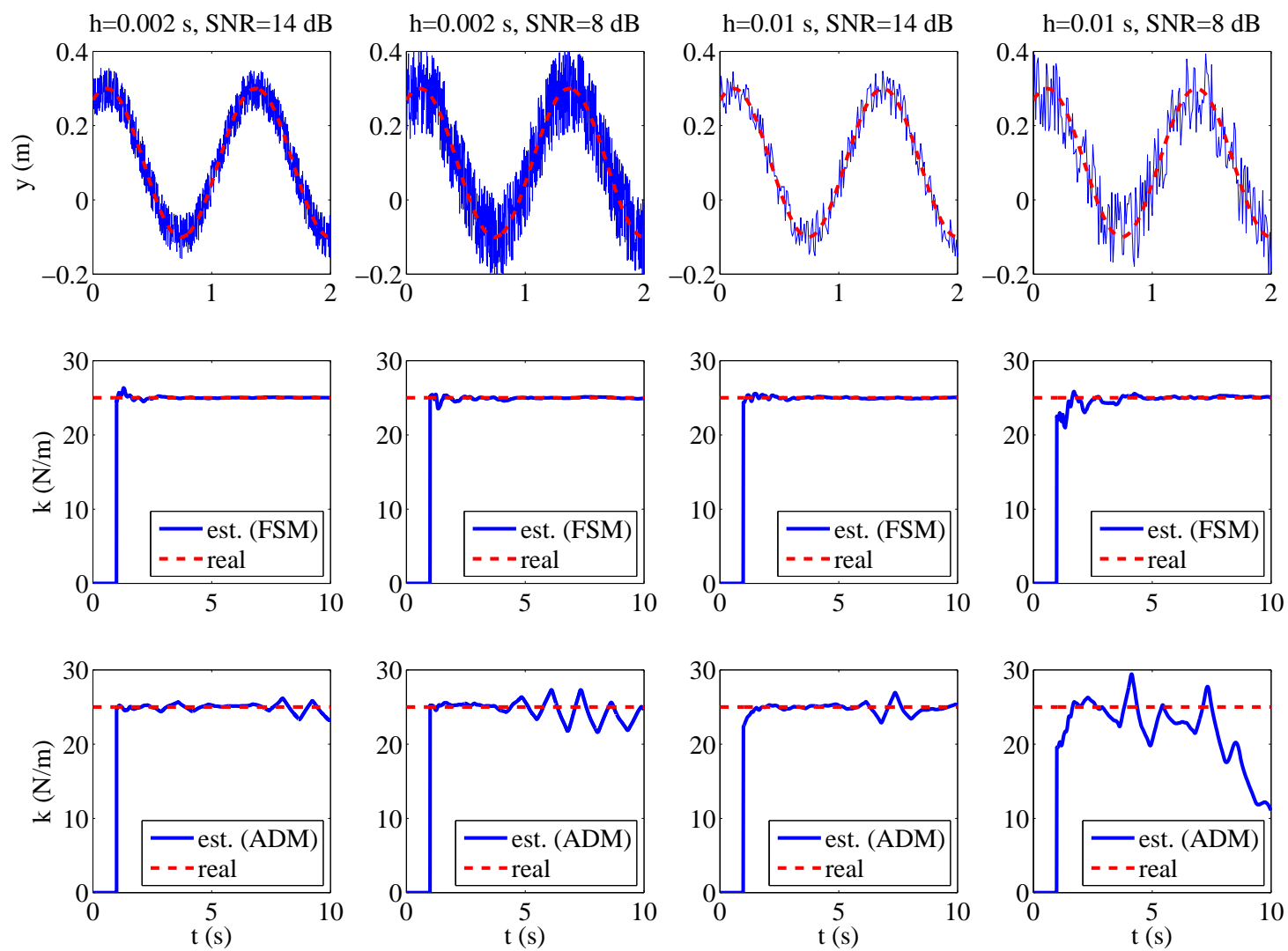

Figure 5: The comparison of ADM and FSM-based estimators in the case with measurement noise for different values of signal-to-noise rate $S N R$ and sampling time $h$.

Table 1: The mean absolute percentage error for ADM and FSM-based estimators, depending on the signalto-noise rate and sampling time.

\begin{tabular}{|c|cc|cc|}
\hline & MAPE (\%) for ADM & MAPE (\%) for FSM & \\
\hline$h$ & $S N R=23.62$ & $\begin{array}{c}S N R=3.48 \\
(\mathrm{~dB})\end{array}$ & $\begin{array}{c}S N R=23.62 \\
(\mathrm{~dB})\end{array}$ & $\begin{array}{c}S N R=3.48 \\
(\mathrm{~dB})\end{array}$ \\
\hline 0.002 & 0.547 & 6.306 & 0.150 & 1.467 \\
0.005 & 0.906 & 10.85 & 0.242 & 2.336 \\
0.01 & 1.499 & 17.16 & 0.368 & 3.617 \\
0.02 & 2.493 & 24.39 & 0.738 & 6.102 \\
\hline
\end{tabular}



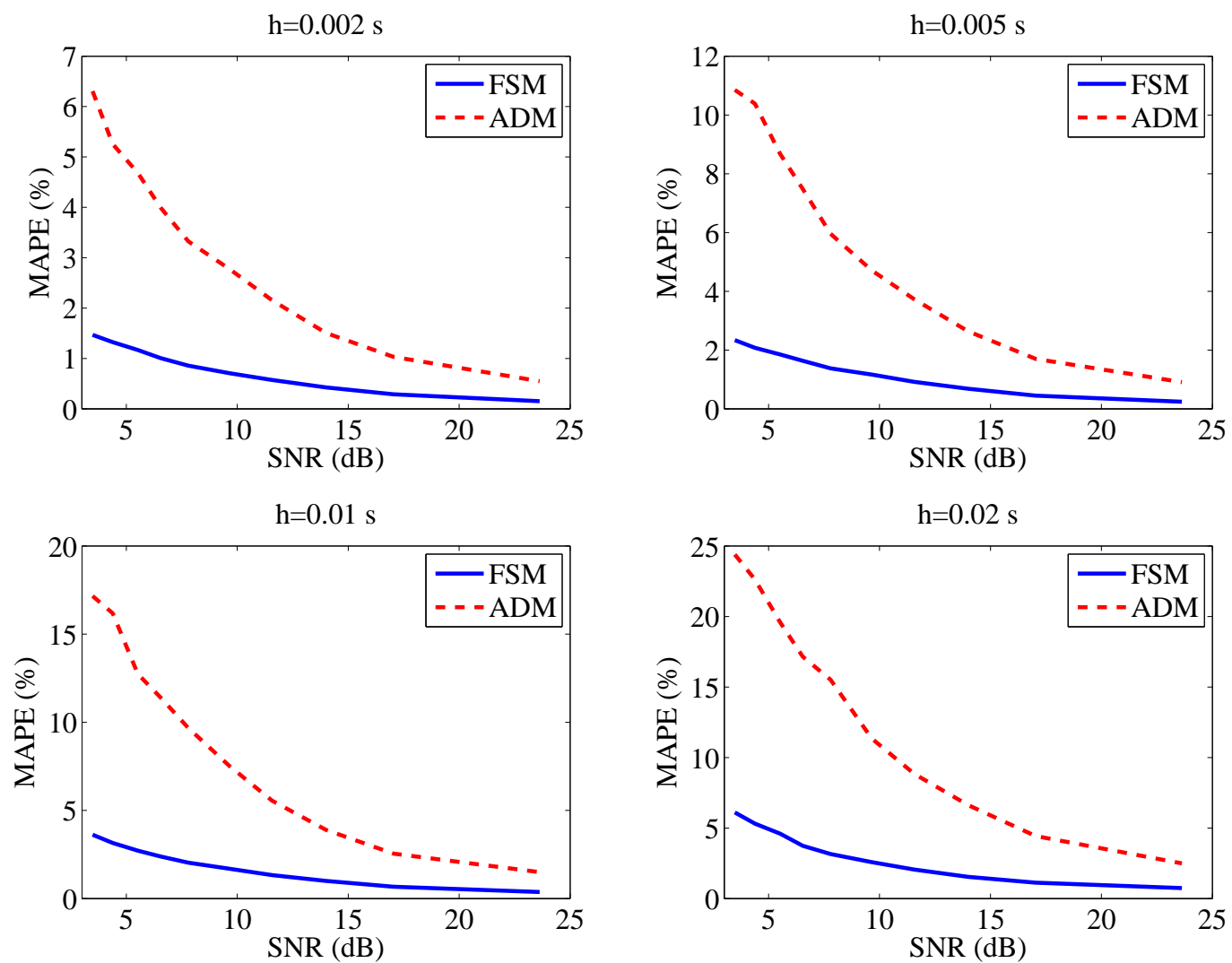

Figure 6: The mean absolute percentage error $M A P E$ depending on the signal-to-noise rate $S N R$ and sampling time $h$, for ADM and FSM-based estimators. 
Table 2: Estimated values and ARE of system parameters in the cases with and without measurement noise.

\begin{tabular}{c|c|cc|cc}
\hline & & Without noise (Fig. 7) & With noise (Fig. 8) & \\
\hline & Real & Estimated & ARE & Estimated & ARE \\
& value & value & $(\%)$ & value & $(\%)$ \\
\hline$m$ & 1.8754 & 1.8750 & 0.0194 & 1.8688 & 0.3494 \\
$c$ & 3.7367 & 3.7274 & 0.2498 & 3.7206 & 0.4305 \\
$k$ & 377.2706 & 377.1791 & 0.0241 & 375.1733 & 0.5558 \\
\hline
\end{tabular}

input step force is $u=4 \mathrm{~N}$, [19]. Initial conditions are $y(0)=y_{0}=0.4 \mathrm{~m}$ and $\dot{y}(0)=v_{0}=0.3$ $\mathrm{m} / \mathrm{s}$. The sampling time is $10^{-3} \mathrm{~s}$.

The Laplace transform of the (59) is

$$
m\left(s^{2} y(s)-s y_{0}-v_{0}\right)+c\left(s y(s)-y_{0}\right)+k y(s)=u(s),
$$

where the initial conditions $y_{0}$ and $v_{0}$ are unknown. By comparing (78) with Eq. (3) it follows that

$$
a_{0}=\frac{k}{m}, \quad a_{1}=\frac{c}{m}, \quad b_{0}=\frac{1}{m}, \quad c_{0}=v_{0}+\frac{c}{m} y_{0}, \quad c_{1}=y_{0} .
$$

By applying algebraic parameter identification algorithm described in Section 3.1, we can identify parameters $a_{0}, a_{1}$ and $b_{0}$, and finally calculate the system parameters: $m=1 / b_{0}$, $c=a_{1} / b_{0}$ and $k=a_{0} / b_{0}$.

The following parameters of the estimator are used in the simulation: frequency shift $q=0.3$, invariant filter order $\bar{n}=5$, and filter poles are $\{-10.0,-10.5,-11.0,-11.5,-12.0\}$. The estimated values and ARE of the system parameters in the cases with and without measurement noise, at the end of the time interval $T=1 \mathrm{~s}$, are listed in Table 2 .

Fig. 7 shows the input force, output position and estimated parameters in the case without measurement noise. We can see that in the time instant $\varepsilon=0.5 \mathrm{~s}$ when the estimator is switched on, the convergence toward real values of the system parameters is almost instantaneous.

Fig. 8 shows the input force, output position and estimated parameters in the case with measurement noise. The noise signal is generated by means of piece-wise constant random variables uniformly distributed in the interval $[-0.01,0.01]$ for the output position (about $10 \%$ of the maximum amplitude), and in the interval $[-0.2,0.2]$ for the input force (about $5 \%$ of the maximum amplitude). We can see that, after short transient, the estimator converges toward real values of system parameters.

\subsection{Example 3: Identification of the frequencies of the exogenous periodic perturbation}

The following example is the problem of determination of frequencies of the exogenous perturbation containing two harmonic signals with different frequencies

$$
f(t)=A_{1} \sin \left(\omega_{1} t+\phi_{1}\right)+A_{2} \sin \left(\omega_{2} t+\phi_{2}\right) .
$$



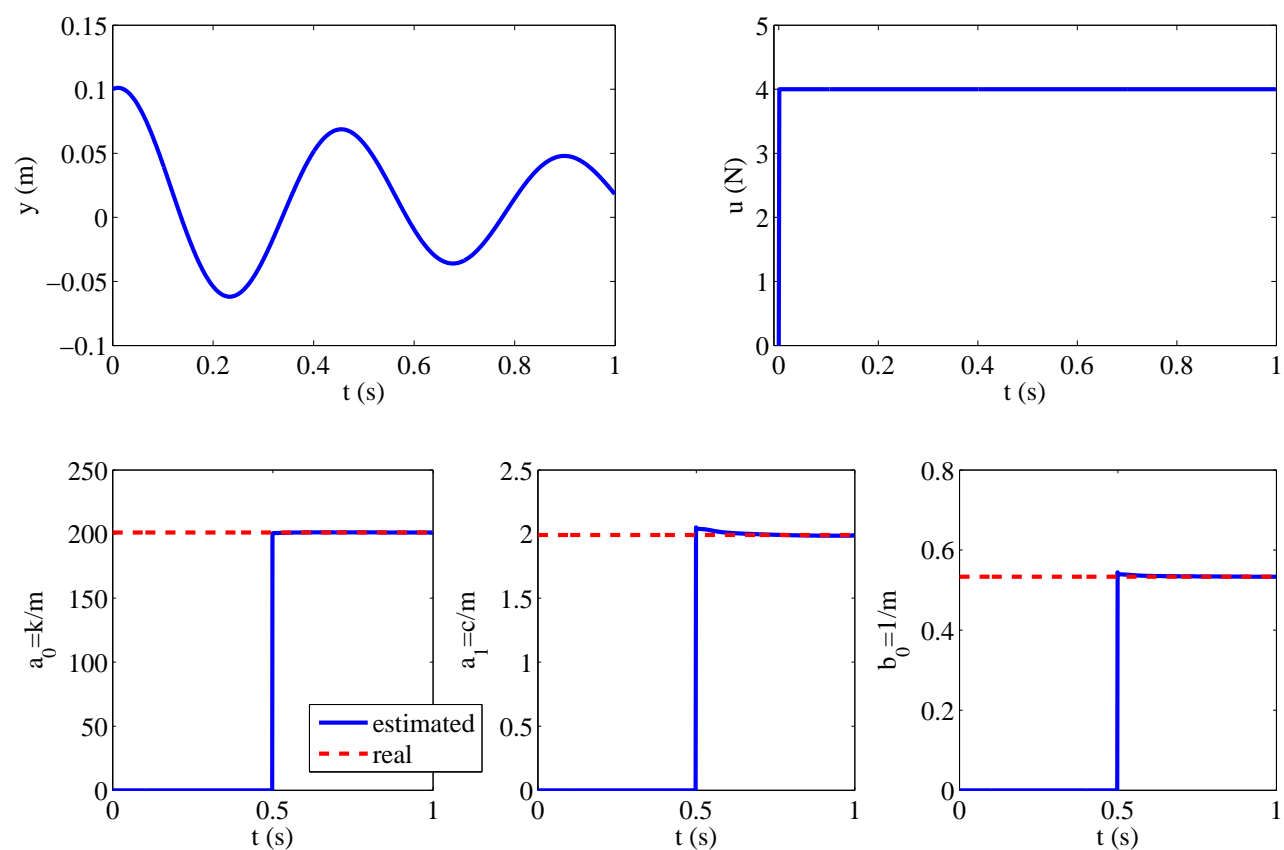

Figure 7: The system input, output and estimated parameters in the case without measurement noise.

All the signal parameters are unknown. In the case when $u=0$, the Laplace transform of the $(59)$ is

$$
m\left(s^{2} y(s)-s y_{0}-v_{0}\right)+c\left(s y(s)-y_{0}\right)+k y(s)=\sum_{i=1}^{2}\left(\frac{A_{i} \omega_{i} \cos \left(\phi_{i}\right)}{s^{2}+\omega_{i}^{2}}+\frac{s A_{i} \omega_{i} \sin \left(\phi_{i}\right)}{s^{2}+\omega_{i}^{2}}\right),
$$

Multiplying above expression with $\left(s^{2}+\omega_{1}^{2}\right)\left(s^{2}+\omega_{2}^{2}\right)$ and after some manipulation, we get

$$
\left(s^{6}+\sum_{i=0}^{5} a_{i} s^{i}\right) y(s)=\sum_{i=0}^{5} c_{i} s^{i}
$$

where the coefficients $c_{i}, i=0,1, \ldots, 5$, depend on initial conditions and amplitudes and phases of the exogenous signal, while the coefficients $a_{i}, i=0,1, \ldots, 5$, depend on system parameters and frequencies of the signal:

$$
\begin{gathered}
a_{0}=k \omega_{1}^{2} \omega_{2}^{2}, \quad a_{1}=c \omega_{1}^{2} \omega_{2}^{2}, \quad a_{2}=k\left(\omega_{1}^{2}+\omega_{2}^{2}\right)+\omega_{1}^{2} \omega_{2}^{2}, \\
a_{3}=c\left(\omega_{1}^{2}+\omega_{2}^{2}\right), \quad a_{4}=k+\omega_{1}^{2}+\omega_{2}^{2}, \quad a_{5}=c .
\end{gathered}
$$

The frequencies can be determined from the following equations

$$
\omega_{1}^{4}-\frac{a_{3}}{a_{5}} \omega_{1}^{2}+\frac{a_{1}}{a_{5}}=0, \quad \omega_{2}^{2}=\frac{a_{3}}{a_{5}}-\omega_{1}^{2} .
$$



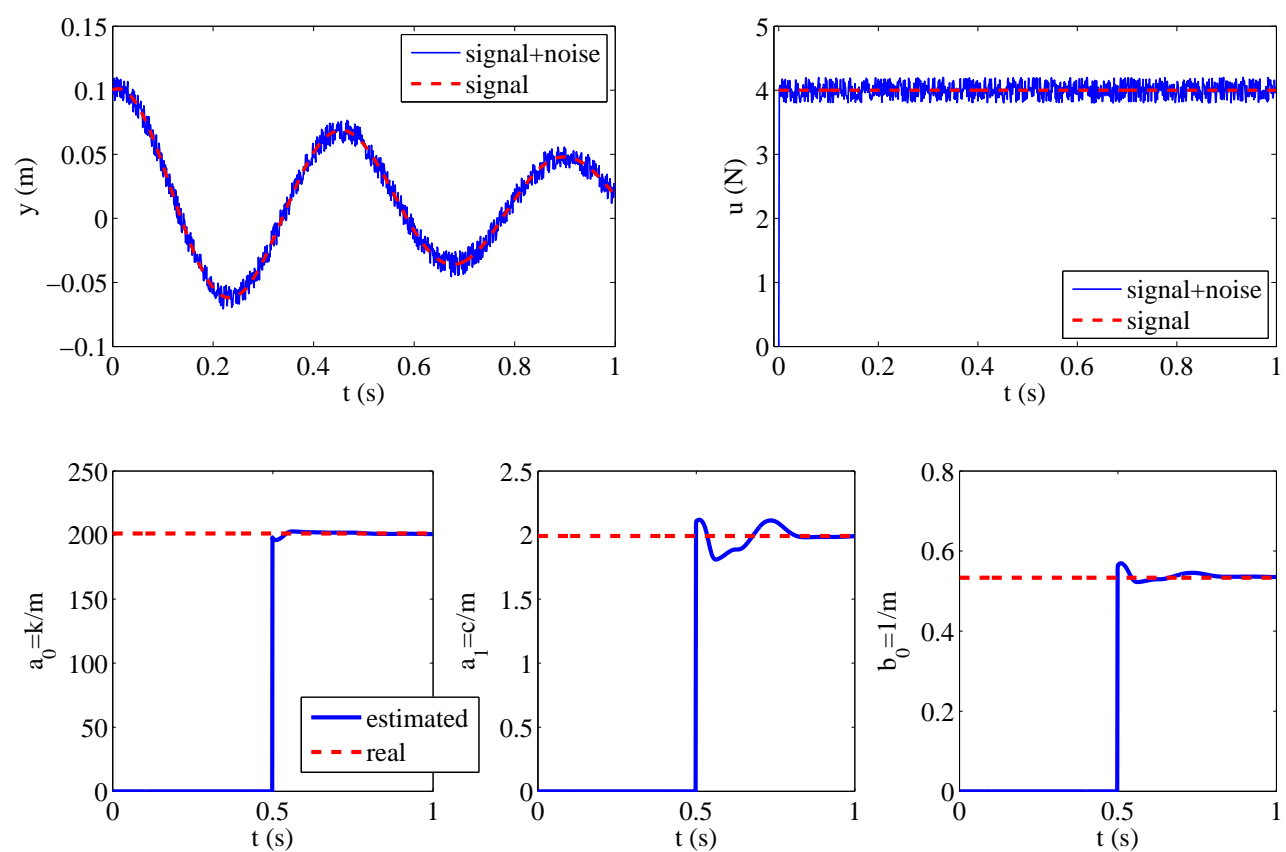

Figure 8: The system input, output and estimated parameters in the case with measurement noise.

By applying the algebraic parameter identification algorithm, we can identify the parameters $a_{i}, i=0,1, \ldots, 5$. The following parameters of the estimator are used in the simulation: the frequency shift $q=0.3$, the invariant filter order $\bar{n}=7$, and the filter poles are

$\{-3.0,-3.6,-4.3,-5.0,-5.6,-6.3,-7.0\}$.

Fig. 9 shows the estimated parameters in the case when the system and signal parameters are: $m=1 \mathrm{~kg}, c=4 \mathrm{Ns} / \mathrm{m}, k=13 \mathrm{~N} / \mathrm{m}, \omega_{1}=9 \mathrm{rad} / \mathrm{s}, \omega_{2}=11 \mathrm{rad} / \mathrm{s}, A_{1}=0.05 \mathrm{~N}$, $A_{2}=0.06 \mathrm{~N}, \phi_{1}=0.1$ and $\phi_{2}=0.2$.

The obtained relative errors of the parameters $a_{i}, i=0,1, \ldots, 5$ at the end of time interval $t=4 \mathrm{~s}$ are $0.0008 \%, 0.0013 \%, 0.0013 \%, 0.0045 \%, 0.0014 \%, 0.0057 \%$, respectively.

\section{Conclusions}

In this paper an alternative to algebraic derivative-based parameter identification of the linear SISO systems is proposed. The proposed approach is based on the difference and the shift operators and corresponding frequency-shifting property of the Laplace transform. The main benefit of this approach is stable state-space filter realization of the estimator. The simulation results demonstrate fast, almost instantaneous, convergence of the estimated parameters toward true values of system parameters. It is shown that the proposed estimator is insensitive to unknown initial conditions and robust with respect to measurement noise. The proposed methodology can be applied also for the stable filter realization of the algebraic 

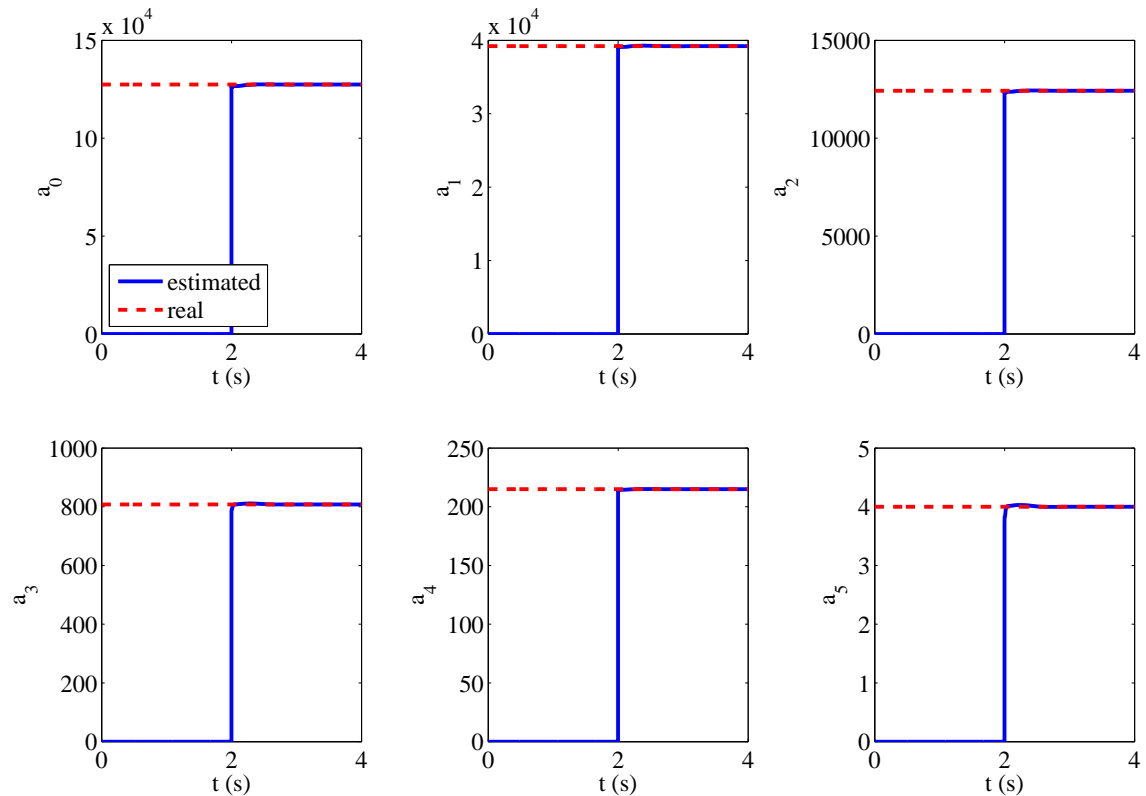

Figure 9: The estimated parameters in the case of the periodic disturbances.

state estimators and the signal derivative estimators. The future work will be oriented toward applications of the proposed approach to the identification and estimation methods in the feedback control of distributed parameter systems.

\section{Acknowledgment}

This work has been supported by Croatian Science Foundation under the project 9354 Control of Spatially Distributed Dynamical Systems.

\section{References}

[1] R. Isermann, M. Mnchhof, Identification of Dynamic Systems: An Introduction with Applications, Springer-Verlag, 2011.

[2] A. Deb, S. Roychoudhury, G. Sarkar, Analysis and Identification of Time-Invariant Systems, TimeVarying Systems, and Multi-Delay Systems using Orthogonal Hybrid Functions: Theory and Algorithms with MATLAB, Springer-Verlag, 2016.

[3] X. Ma, F. Ding, Recursive and iterative least squares parameter estimation algorithms for observability canonical state space systems, Journal of the Franklin Institute 352 (1) (2015) 248-258.

[4] X. Wang, F. Ding, Performance analysis of the recursive parameter estimation algorithms for multivariable box-jenkins systems, Journal of the Franklin Institute 351 (10) (2014) 4749-4764.

[5] M. Fliess, H. Sira-Ramírez, An algebraic framework for linear identification, ESAIM Control Optimisation and Calculus of Variations 9 (1) (2003) 151-168.

[6] M. Fliess, H. Sira-Ramírez, Closed-loop parametric identification for continuous-time linear systems via new algebraic techniques, in: H. Garnier, L. Wang (Eds.), Identification of continuous-time models from sampled data, Advances in Industrial Control, Springer, 2008, pp. 363-391. 
[7] J. Mikusinski, Operational Calculus, 2nd Edition, Pergamon Pr, 1984.

[8] A. P. Prudnikov, K. Skórnik, Operational calculus and related topics, Chapman \& Hall/CRC, 2006.

[9] J. R. Trapero, H. Sira-Ramírez, V. F. Batlle, An algebraic frequency estimator for a biased and noisy sinusoidal signal, Signal Processing 87 (6) (2007) 1188-1201.

[10] J. R. Trapero, H. Sira-Ramírez, V. Feliu Batlle, On the algebraic identification of the frequencies, amplitudes and phases of two sinusoidal signals from their noisy sum, International Journal of Control 81 (3) (2008) 507-518.

[11] M. Mboup, Parameter estimation for signals described by differential equations, Applicable Analysis 88 (1) (2009) 29-52.

[12] M. Fliess, C. Join, H. Sira-Ramirez, Non-linear estimation is easy, Int. J. Modelling Identification and Control 4 (1) (2008) 12-27.

[13] R. Morales, E. Segura, J. Somolinos, L. Núñez, H. Sira-Ramírez, Online signal filtering based on the algebraic method and its experimental validation, Mechanical Systems and Signal Processing 66-67 (2016) 374-387.

[14] M. Mboup, C. Join, M. Fliess, Numerical differentiation with annihilators in noisy environment, Numerical Algorithms 50 (4) (2009) 439-467.

[15] K. I. Taarit, L. Belkoura, M. Ksouri, J.-P. Richard, A fast identification algorithm for systems with delayed inputs, International Journal of Systems Science 42 (3) (2011) 449-456.

[16] L. Belkoura, J.-P. Richard, M. Fliess, Parameters estimation of systems with delayed and structured entries, Automatica 45 (5) (2009) 1117-1125.

[17] H. Sira-Ramírez, C. G. Rodríguez, J. C. Romero, A. L. Juárez, Algebraic Identification and Estimation Methods in Feedback Control Systems, John Wiley \& Sons, 2014.

[18] R. Delpoux, T. Floquet, On-line parameter estimation via algebraic method: An experimental illustration, Asian Journal of Control 17 (1) (2015) 315-326.

[19] F. Beltrán-Carbajal, G. Silva-Navarro, Adaptive-like vibration control in mechanical systems with unknown paramenters and signals, Asian Journal of Control 15 (6) (2013) 1613-1626.

[20] C. Garcia-Rodríguez, J. Cortés-Romero, H. Sira-Ramírez, Algebraic identification and discontinuous control for trajectory tracking in a perturbed 1-DOF suspension system, IEEE Transactions on Industrial Electronics 56 (9) (2009) 3665-3674.

[21] R. Garrido, A. Concha, An algebraic recursive method for parameter identification of a servo model, IEEE/ASME Transactions on Mechatronics 18 (5) (2013) 1572-1580.

[22] A. Gensior, J. Weber, J. Rudolph, H. Guldner, Algebraic parameter identification and asymptotic estimation of the load of a boost converter, IEEE Transactions on Industrial Electronics 55 (9) (2008) $3352-3360$.

[23] T. Liu, F. Gao, Robust step-like identification of low-order process model under nonzero initial conditions and disturbance, IEEE Transactions on Automatic Control 53 (11) (2008) 2690-2695.

[24] R. V. Churchill, Operational mathematics, 3rd Edition, McGraw-Hill, 1972.

[25] M. Fliess, M. Mboup, H. Mounier, H. Sira-Ramirez, Questioning some paradigms of signal processing via concrete examples, in: H. Sira-Ramirez, G. Silva-Navarro (Eds.), Algebraic Methods in Flatness, Signal Processing and State Estimation, Editorial Lagares, 2003, pp. 1-21. 